\title{
USO DE ANTI-INFLAMATÓRIOS EM ATLETAS E SOLUÇÕES RELACIONADAS A DIETOTERAPIA E FITOTERAPIA: UMA REVISÃO ${ }^{1}$
}

\author{
USE OF ANTI-INFLAMMATORIES FOR ATHLETES AND SOLUTIONS \\ RELATED TO DIETOTHERAPY AND PHYTOTHETARY: A REVIEW
}

\section{Bruno César Thomazi Freitas Pezzini², Gabriela Maestri², Geneci Santos de Jesus Reis ${ }^{2}$ Giovana Vechi ${ }^{3}$}

\section{RESUMO}

Devido ao seu efeito analgésico, antipirético e anti-inflamatório, muitos atletas fazem o uso de anti-inflamatórios, sendo os mais utilizados os do tipo não-esteroidais (AINEs), para o tratamento da dor aguda proveniente da inflamação. Os efeitos colaterais são bastante comuns no caso dos AINEs (dispepsia, náuseas, vômitos, lesões gastrointestinais e reações cutâneas), sendo o uso de alimentos com potencial anti-inflamatório e antioxidante uma alternativa menos agressiva aos medicamentos e em geral, sem efeitos adversos. Visto que o consumo de anti-inflamatórios e a automedicação em atletas deve ser desencorajado por profissionais da saúde, o objetivo desta revisão foi analisar o uso de medicamentos anti-inflamatórios na prática esportiva e revisar alternativas de tratamento não medicamentoso no processo inflamatório. Nutrientes e fitoterápicos como curcumina, hesperidina, alcaçuz, espinheira-santa, pequi, cereja azeda, ômega 3, resveratrol, chá verde, licopeno, quercetina e gengibre, tem sido amplamente estudados devido a sua atividade anti-inflamatória. Desta forma, conclui-se que estes alimentos e compostos bioativos podem ser uma alternativa para o controle da inflamação causada pelo exercício físico.

Palavras-chave: Alimentos funcionais; Exercício físico; Inflamação.

\section{ABSTRACT}

Due to its analgesic, antipyretic and anti-inflammatory effect, many athletes make the use of antiinflammatory drugs, the most used being non-steroidal (NSAIs) for the treatment of acute pain induced by inflammation. Side effects are quite common in the case of NSAIs (dyspepsia, nausea, vomiting, gastrointestinal lesions, skin reactions), and the use of foods with anti-inflammatory potential and antioxidant is a less aggressive alternative to medications, without adverseeffects. Since the use of anti-inflammatory drugs and self-medication in athletes should be discouraged by health professionals, the aim of this review was to analyze the use of anti-inflammatory drugs in sports practice and to review non-drug treatment alternatives in the inflammatory process. Nutrients and bioactive compounds such as curcumin, hesperidine, licorice, blackhead, pequi, sour cherry, omega 3, resveratrol, green tea, lycopene, quercetin and ginger, has been widely studied due to its anti-inflammatory activity. Thus, it is concluded that these functional foods and bioactive compounds may be an alternative to control inflammation caused by physical exercise.

Keywords: Exercise; Functional foods; Inflammation.

\footnotetext{
${ }^{1}$ Trabalho de Conclusão de Estágio.

2 Acadêmicos do Curso de Nutrição - Universidade do Vale do Itajaí - UNIVALI. E-mail: bepezzini@gmail.com; gaabi_maestri@hotmail.com; telesgeneci@gmail.com.

${ }^{3}$ Orientadora e Professora - Curso de Nutrição - Universidade do Vale do Itajaí - UNIVALI. E-mail: giovanavechi@univali.br
} 


\section{INTRODUÇÃO}

O esforço físico decorrente da prática esportiva desencadeia danos musculares em atletas, podendo levar a desgastes do organismo como o aumento da inflamação. Os atletas apresentam aumento dos indicadores de lesões musculares e marcadores inflamatórios logo após o esforço físico extenuante, o que ilustra o efeito agudo sobre a estrutura muscular (PUGGINA et al., 2016).

O exercício físico extenuante é acompanhado também pela produção de radicais livres, devido a oxidação celular, desencadeando um desequilíbrio entre o ataque oxidativo e o sistema de defesa antioxidante, ocasionando uma lesão acompanhada por um processo inflamatório ao nível das fibras musculares, geralmente causada por excesso de treino (overtraining) ou por recuperação inadequada. Este quadro conduz a uma redução da função muscular associado a fadiga, redução da força, alterações histológicas evidentes e dores musculares, além da perda do rendimento esportivo (ANDRADE; MARREIRO, 2011; PANKANIN, 2018).

Em decorrência deste processo, muitos atletas utilizam medicamentos anti-inflamatórios e relaxantes musculares a fim de tratar lesões decorrentes do esforço físico. No Brasil, os anti-inflamatórios como: Paracetamol, AAS, Dipirona Diclofenaco, Ibuprofeno, Nimesulida são encontrados facilmente em farmácias podendo ser adquiridos sem prescrição médica (SILVA et al., 2016).

Os anti-inflamatórios não-esteroidais (AINEs) constituem uma das classes de fármacos mais consumidas no mundo. Possuem efeitos analgésicos, antipiréticos e anti-inflamatórios que são utilizados no tratamento da dor aguda proveniente da inflamação. Seus efeitos ocorrem através da redução da enzima ciclooxigenase (COX), resultando na diminuição dos precursores de prostaglandinas e tromboxanos (SILVA et al., 2016). Os efeitos colaterais mais comuns são: dispepsia, náuseas, vômitos, lesões gastrointestinais, reações cutâneas, insuficiência renal reversível, entre outros (GOMES et al., 2019; LARINI, 2008).

O uso de alimentos com potencial anti-inflamatório e antioxidante traz benefícios na recuperação de atletas e vem sendo estudado como uma alternativa não medicamentosa, pois são menos agressivos e em geral, não apresentam efeitos colaterais (PANAHI et al., 2014). Uma alimentação equilibrada e direcionada é de grande relevância na prática esportiva, podendo prevenir ou até mesmo tratar lesões. De acordo com Andrade e Marreiro (2011), alguns alimentos apresentam capacidade de atuarem de forma eficaz no controle da inflamação, por meio da redução das citocinas pró-inflamatórias ou no auxílio da defesa antioxidante.

Diante do exposto, objetiva-se com este trabalho analisar o uso de medicamentos na prática esportiva e demonstrar alternativas de tratamento não medicamentoso no processo inflamatório. 


\section{MATERIAL E MÉTODOS}

O presente trabalho foi uma revisão criteriosa da literatura a partir de artigos científicos nacionais e internacionais, todos indexados entre os anos de 2000 e 2019, priorizando os artigos mais atuais que tratassem sobre o assunto, além de livros e capítulos sobre o tema. Foram utilizadas as bases de dados Pubmed, Google Acadêmico e Scielo para a busca das referências citadas. As palavras-chave utilizadas foram: anti-inflamatórios, prostaglandinas, esporte, atletas, exercício físico, inflamação, AINEs e COX-2 nos idiomas inglês, português e espanhol. Foram incluídos artigos originais e de revisão bibliográfica.

\section{DESENVOLVIMENTO}

\section{ANTI-INFLAMATÓRIOS}

Os principais anti-inflamatórios utilizados por atletas são os anti-inflamatórios não-esteroidais (AINEs) e os esteroidais. Os AINEs estão entre os compostos mais consumidos na terapêutica e como automedicação, mesmo que nenhum seja considerado ideal no controle dos sinais e sintomas que ocorrem nos processos inflamatórios, principalmente aos relacionados com as articulações (LARINI, 2008). Entre os mais utilizados estão o Ácido Acetilsalicílico (AAS), Paracetamol, Dipirona, Diclofenaco, Ibuprofeno e a Nimesulida (SILVA et al., 2019).

Os AINEs são medicamentos com ação anti-inflamatória, antitérmica, analgésica e antitrombótica, que atuam inibindo a ciclooxigenasel (COX1) e ciclooxigenase2 (COX2). Em inflamações, a COX2 tem a função de sintetizar prostaglandinas com ação pró-inflamatória que ativam nociceptores que aumentam a sensação de dor e a COX1 sintetiza prostaglandinas com função de proteção tecidual (CORREA; BARONI; CADORE, 2010). Nesse sentido, os AINEs atuam inibindo a cascata do ácido araquidônico, de maneira seletiva ou não, impedindo a formação de prostaglandinas (BURUKOGLU et al., 2016).

Este mecanismo gera diminuição da sensibilização das terminações nervosas nociceptivas e mediadores inflamatórios como a bradicinina e serotonina e provável alívio da cefaleia, motivado por redução da vasodilatação. O efeito antipirético acontece a partir da diminuição de prostaglandinas produzidas em resposta ao pirógeno inflamatório, a interleucina-1, que é responsável pela elevação do ponto de ajuste hipotalâmico para o controle da temperatura da febre (LARINI, 2008).

O uso de AINEs apresenta efeitos adversos como lesões na mucosa gastrointestinal. No estômago, em pH ácido, eles atravessam a mucosa por ação do pH citoplasmático próximo a neutralidade, se ionizam e, impossibilitados de saírem do interior celular, se acumulam e provocam retrodifusão de íons $\mathrm{H}+$ para o lúmen, ocasionando edema e hemorragia na região (GOMES et al., 2019). Os efeitos colaterais mais importantes resultam do bloqueio da produção de prostaglandinas no estômago. 
Essas prostaglandinas atuam como agentes citoprotetores da mucosa, inibindo a secreção ácida pelo estômago, aumentando o fluxo sanguíneo local e a secreção de muco protetor. Sendo que o tratamento em longo prazo pode ocasionar erosões e úlceras gástricas e duodenais (OLIVEIRA et al., 2019).

Nesse sentido, os AINEs possuem a capacidade de interagir com a camada de muco e com a bicamada fosfolipídica do trato gastrointestinal, tornando o revestimento da mucosa menos hidrofóbico e mais exposto a agentes agressores como os ácidos e as pepsinas presentes no estômago e a bílis no intestino delgado, além de dissociar a cadeia de fosforilação oxidativa mitocondrial, conduzindo uma série de eventos que provocam a depleção de adenosina trifosfato (ATP) e a perda de integridade das junções intercelulares do trato gastrointestinal, aumentando a permeabilidade da mucosa, aumentando crescimento de bactérias gram negativas e dificultando a digestão e absorção de nutrientes, consequentemente alterando o estado nutricional dos indivíduos (VILELA, 2019).

Os anti-inflamatórios esteroidais derivam do colesterol a partir da sintetização do córtex supra-renal, também denominados de hormônios adrenocortioides, adrenocorticosteroides ou simplesmente corticosteroides. Essas substâncias são liberadas na circulação, exercendo efeitos importantes sobre o metabolismo intermediário (glicocorticoides) e na retenção de sal (mineralocorticóides), enquanto outros possuem atividade androgênica ou estrogênica. Sendo os principais glicocorticóides anti-inflamatórios são a beclometasona, budenosida, flunisolida e a fluticasona (LUCAS et al., 2019).

A corticotropina e os esteroides que tem atividade glicocorticoide são utilizados no tratamento de numerosos distúrbios inflamatórios e alérgicos. Os glicocorticoides sintéticos produzem efeitos anti-inflamatórios por meio da inibição da ação enzimática da fosforilase A2, que impede a liberação do ácido araquidônico e por consequência a formação das prostaglandinas, de tromboxanos e leucotrienos (SILVA et al., 2019; LARINI, 2008).

Os principais efeitos adversos da utilização de anti-inflamatórios esteroidais incluem sua ação hormonal, pois podem gerar a chamada síndrome de Cushing iatrogênica (por causas exógenas) e complicações como úlceras pépticas, miopatia, tontura e candidíase orofaríngea (SILVA et al., 2019; LARINI, 2008).

Desta forma Corrigan e Kaslauskas (2003), descreveram que o consumo desenfreado de AINEs por atletas eram por lesões decorrentes por demanda especifica do esporte em questão, insuficiência do período requerido para a recuperação da lesão com uso repetitivo da área anatômica lesada, condições biomecânicas impróprias, atletas com lesões de menor extensão que não alteram sua carga de treinamento e fazem uso crônico da droga.

Como efeitos adversos em atletas podemos destacar o estudo realizado por Mcanulty e colaboradores (2007) que investigaram a ação do ibuprofeno nos indicadores de estresse oxidativo onde foi demonstrado significativo aumento de concentração de indicadores de estresse oxidativo no plasma e urina dos atletas pós competição. 
A partir do exposto, Silva e colaboradores (2016) avaliaram o padrão de consumo de medicamentos de 71 (100\%) atletas universitários, sendo que 38 atletas (53,5\%) referiram utilizar medicamentos para tratamento de gripes e resfriados, 37 (52,1\%) por dores musculares e $25(35,2 \%)$ por presença de inflamações. Dentre os atletas, 35 (49,3\%) afirmaram se automedicar para tratar os sintomas citados. Entre os principais medicamentos utilizados destacam-se os AINEs, analgésicos, relaxantes musculares e antigripais.

Rose e colaboradores (2006) avaliaram 234 atletas de 25 esportes diferentes e observaram que 44\% dos atletas informaram no controle de dopping a utilização de medicamentos até três dias antes da competição, sendo 58 (36,9\%) atletas referindo o uso de AINEs, 37 (23,6\%) analgésicos, 10 (6,4\%) antibióticos, 7 (4,5\%) antigripais e 45 (26,8\%) outros medicamentos. Os autores concluíram que há uso exacerbado de anti-inflamatórios e analgésicos em várias modalidades desportivas, causando preocupação em termos de controle de sintomas de atletas em competição.

Correa e colaboradores (2013), também analisaram 12 atletas de força que realizaram o treino após a ingestão de ibuprofeno com o objetivo de verificar a melhora do desempenho no treino de força, porém não foram verificadas diferenças no desempenho do grupo que ingeriu o anti-inflamatório do grupo placebo. A inibição da síntese de prostaglandinas tem repercussão negativa sobre a síntese proteica induzida por sessão de treino de força.

Para o processo de hipertrofia após a sessão de treinamento, faz-se necessário a produção de espécies reativas de oxigênio (EROs), para que ocorra a indução da resposta adaptativa ao treinamento e o uso de fármacos que desempenham a função anti-inflamatória pode trazer prejuízos quando ingeridos perto do horário de treinamento, diferente de alimentos com antioxidantes não isolados, que trazem benefícios ao atleta (SILVA; CHAVES, 2015). Os AINEs utilizados como forma de supressão da dor muscular, acabam por prejudicar a regeneração da lesão de origem da atividade esportiva de alta intensidade, já que podem ocorrer efeitos adversos na rigidez e contração muscular e na sua regeneração (XAVES et al., 2017).

\section{PROBLEMAS GERADOS PELO USO DE ANTI-INFLAMATÓRIOS: ALTERAÇÕES GASTROINTESTINAIS}

AINEs, como o ácido acetil salicílico (AAS) por exemplo, funcionam através da inibição irreversível das enzimas ciclooxigenase 1 e 2 (COX-1 e COX-2) que desempenham papel na geração da inflamação, porém, com inibição da COX-1, a síntese de prostaglandinas gástricas que funcionam como citoprotetores da mucosa estomacal é impedida, levando a produção de maior quantidade de secreção ácida e diminuição do fluxo sanguíneo na mucosa, causando úlceras gástricas ou duodenais (GILMAN; GOODMAN, 2010; BRENOL et al., 2000).

Funções fisiológicas importantes são atribuídas à COX-1 através da síntese de prostanóides, como a manutenção da homeostase, proteção da mucosa gástrica e agregação plaquetária, que acabam por serem inibidas devido ao uso de AINEs (MORO et al., 2019). Reações comuns ao uso 
indiscriminado de AINEs incluem hipersensibilidade, dependência física e psíquica, encobrimento dos sintomas e agravamento de doenças de base (OLIVEIRA; PELÓGIA, 2011).

Devido aos danos causados pelos AINES no epitélio intestinal, o mesmo tem comprometida a função de barreira contra substâncias nocivas, implicando em dificuldade na permeabilidade intestinal tendo como consequência a disbiose (KERCKHOFFS, 2010). Tal desequilíbrio entre bactérias protetoras e agressoras ocasiona vulnerabilidade a diversas enfermidades e deficiência do sistema imunológico com aumento de microorganismos patogênicos e inflamações, levando o atleta ao ciclo vicioso de uso de anti-inflamatórios (CHANG; LIN, 2016).

Alterações na microbiota intestinal também tem sido associada às características do estado nutricional como eutrofia e obesidade, sendo que o excesso de peso é um grande responsável pela colonização com bactérias maléficas (MILLION; LAGIER; YAHAV; PAUL, 2013).

\section{DANOS HEPÁTICOS}

Doses elevadas de AINEs podem acarretar em danos hepáticos. Um exemplo prático é o uso de doses acima de $4 \mathrm{~g} /$ dia de paracetamol que levam o fármaco a um processo oxidativo que libera $\mathrm{o}$ n-acetil-p-benzoquinonamina, um metabólito tóxico que causa lesão hepática irreversível (BUSHEL et al., 2007; HE, 2011).

Problemas hepáticos têm sido relatados em diversos países relacionados ao uso de nimesulida, associando o mesmo a alterações no funcionamento das mitocôndrias que podem levar a morte celular hepática, por meio de apoptose (ARAÚJO, 2012).

Como consequência de doença hepática crônica, pode-se citar a má absorção intestinal de gorduras, hipoglicemia com resistência insulínica, esteatose e hipertrigliceridemia, além de níveis elevados de alguns aminoácidos que trazem agravantes neurológicos (MAIO; DICHI; BURINI, 2000).

Deve-se observar as doses diárias máximas recomendadas na bula do medicamento, como por exemplo no caso do paracetamol cuja prescrição não deve ultrapassar 325mg simultaneamente com outros fármacos que também possuam paracetamol em sua fórmula (BRAYNER; SILVA; ALMEIDA, 2018).

O uso indiscriminado de AINEs por atletas com o objetivo de tratar lesões ou como suposto ajudante na recuperação e desemprenho é pratica bastante comum, sendo pouco o conhecimento destes a respeito das doses recomendadas e dos efeitos colaterais (O'CONNOR et al., 2018; RUDGARD; HIRSCH; COX, 2018).

\section{NUTRIENTES E FITOTERÁPICOS PARA DOR E INFLAMAÇÃO: CURCUMINA}

A curcumina é um pigmento fenólico de cor amarela presente na cúrcuma (Curcuma longa L.), espécie pertencente à família da Zingiberaceae. Este composto é inibidor de diversas vias pró-inflamatórias, 
como por exemplo, NF-K $\beta$ e TNF- $\alpha$. Os estímulos extracelulares que ativam a cascata de formação do NF-אB dependem da presença de espécies reativas de oxigênio, que são decorrentes de lesões e processos inflamatórios (SUETH-SANTIAGO et al., 2015; GRASSO et al., 2017).

Uma pesquisa realizada por Takahashi e colaboradores (2014) com dez homens praticantes de exercício físico suplementados com curcumina via oral $(90 \mathrm{mg})$ ou placebo, 2 horas antes do exercício ou imediatamente após este, mostrou que a suplementação de curcumina, ingerida antes do exercício, reduziu os marcadores de estresse oxidativo, porém quando consumida após o exercício, não se mostrou eficaz.

Drobnic e colaboradores (2014) avaliaram se a curcumina poderia diminuir os danos do estresse oxidativo e inflamação relacionados à lesão muscular aguda causada pelo exercício contínuo intenso. Homens jovens receberam $200 \mathrm{mg}$ de curcumina ou do placebo 1x ao dia. A suplementação foi administrada 48 horas antes de um teste de corrida em declive e permaneceu por 24 horas após o teste (4 dias no total). O grupo da curcumina relatou sentir menos dor nos membros inferiores, porém, essa diferença não apresentou significância estatística. Os marcadores de dano muscular e inflamação tenderam a ser menores no grupo curcumina, mas diferenças significativas foram observadas apenas para a interleucina-8 duas horas após o exercício.

\section{LICOPENO}

O licopeno é um carotenoide de caráter lipossolúvel e está presente nos alimentos de coloração avermelhada e alaranjada, como melancia, mamão, pitanga, goiaba, uva e tomate. O tomate é o vegetal que apresenta maiores concentrações deste nutriente. Sua ação está relacionada a redução da resposta inflamatória e da expressão gênica das enzimas COX-2 e iNOS, e pela diminuição da translocação do fator de transcrição NF-אB do citoplasma para o núcleo (SHAMI; MOREIRA, 2004; MORITZ; TRAMONTE, 2006; NWAICHI; CHUKU; OYIBO, 2015).

O sumo de tomate vermelho, tem sido utilizado por vários atletas profissionais na melhoria da recuperação a nível desportivo por possuir grande biodisponibilidade deste nutriente (cerca de $150 \mathrm{mg}$ de licopeno por litro) (SHAMI; MOREIRA, 2004; PREEDY, 2008).

Tsitsimpikou e colaboradores (2013) avaliaram quinze atletas treinados com níveis basais elevados de lactato desidrogenase $(\mathrm{LDH})$ e creatina fosfoquinase (CPK) detectada durante a avaliação bioquímica de rotina. A amostra foi composta por dois grupos: Grupo tratado que substituiu sua bebida usual de suplementação de carboidratos, consumida regularmente durante e após as sessões de exercício por suco de tomate, referente a $100 \mathrm{~g}$ de tomate e Grupo controle que foram instruídos a continuar o consumo da bebida. Os resultados mostraram redução significativa dos níveis de LDH e $\mathrm{CPK}$, retornando aos níveis quase normais no grupo suplementado com suco de tomate. Ao mesmo tempo, a homocisteína e a proteína $\mathrm{C}$ reativa também foram atenuadas. 


\section{HESPERIDINA}

O flavonoide hesperidina é encontrado em frutas cítricas como laranja e limão, em maiores quantidades na casca. Silva e colaboradores (2019) demonstraram atividade reparadora de lesões gástricas na mucosa já ulcerada, efeito favorecido pela redução dos danos oxidativos da mucosa, devido à redução da migração de neutrófilos e ao fortalecimento da barreira de proteção.

Como protetor gástrico, podemos citar o uso combinado do carotenoide licopeno com a hesperidina já citada anteriormente, sendo o primeiro encontrado em diversas frutas e legumes, especialmente no tomate, cuja biodisponibilidade é aumentada através do aquecimento. $\mathrm{O}$ efeito sinérgico de uma combinação de carotenoide e flavonoide foi relatado devido à sua atividade antioxidante que elimina espécies reativas de oxigênio (EROs) e evita a peroxidação lipídica da membrana (JAIN; KATTI, 2015).

Em concordância, Garg e colaboradores (2001) constataram em seus estudos que a hesperidina possui importantes efeitos anti-inflamatórios e analgésicos. Seu uso pode ser indicado como anti-inflamatório principalmente em pessoas com hipersensibilidade aos AINEs convencionais.

\section{CEREJA AZEDA}

Fonte de polifenóis e antocianinas, que auxiliam na diminuição das dores em atletas e aumentam a recuperação muscular, por reduzir marcadores de estresse oxidativo e inflamação. Para atletas de modalidades de resistência é recomendado o consumo da cereja azeda, pois se trata de um anti-inflamatório natural, sem efeitos colaterais à saúde (VITALE; HUEGLIN; BROAD, 2017).

Bell e colaboradores (2014) examinaram o impacto do concentrado de cereja azeda nos índices fisiológicos de estresse oxidativo, inflamação e danos musculares ao longo de três dias simulados de corrida de bicicleta. A amostra foi composta por ciclistas treinados que tomaram $30 \mathrm{~mL}$ do suplemento duas vezes ao dia, durante sete dias consecutivos. Os resultados mostram que tanto o estresse oxidativo quanto as respostas inflamatórias foram reduzidas com a suplementação. Os autores sugerem que a cereja azeda pode ser eficaz no combate às cascatas oxidativas e inflamatórias pós-exercício contribuindo para a ruptura celular.

\section{ÔMEGA 3}

Os ácidos graxos (AG) Ômega-3 são tipos de gorduras essenciais ao organismo, pois não são sintetizados endogenamente nos seres humanos, devido à carência de enzimas dessaturases. São divididos em ácidos alfa-linolênico (ALA), ácido eicosapentaenóico (EPA) e ácido docosaexaenóico (DHA) (MAHAN; ESCOTT-STUMP, 2012; MARTIN et al., 2006). 
A ingestão de ácidos graxos ômega-3 diminui citocinas pró-inflamatórias além de melhorar a utilização de substratos energéticos e imunidade (LOPEZ-GARCIA et al., 2004; GARÓFOLO; PETRILLI, 2006). No exercício físico, promove diminuição na percepção da dor muscular sendo uma alternativa segura para praticantes de atividade física. As principais fontes alimentares são peixes de águas frias, como cavala, sardinha, salmão, arenque, chia, linhaça e nozes (SANTO et al., 2007).

Garcia e colaboradores (2010), verificaram que ratos suplementados com ômega-3 que realizaram natação, tiveram menos alterações histológicas induzidas pelo exercício. Os autores sugerem um efeito protetor da suplementação além da manutenção da homeostase do sistema muscular e o equilíbrio do sistema oxidante, controlando as respostas inflamatórias, prevenindo lesões no músculo esquelético.

Em concordância, Atashk e colaboradores (2013) objetivaram avaliar o efeito da suplementação de ácidos graxos ômega-3 na dieta sobre os níveis de estresse oxidativo, dano muscular e marcadores inflamatórios após o exercício resistido agudo em atletas jovens, em um delineamento duplo-cego randomizado. A amostra foi composta por vinte indivíduos divididos em grupos iguais que receberam 3000 $\mathrm{mg}$ /dia durante sete dias e foram submetidos a exercícios físicos extenuantes. Os autores concluíram que uma única sessão de exercício intenso leva produção de estresse oxidativo e aumento dos índices de PCR e dano celular em jovens atletas, mas a suplementação de ômega-3 de curto prazo (uma semana) pode melhorar o aumento nesses marcadores, podendo ser uma estratégia eficaz de atenuar o dano oxidativo induzido pelo exercício e inflamação no período pós-exercício.

\section{RESVERATROL}

O resveratrol é um polifenolantioxidante encontrado em muitas plantas, como uvas, nozes e frutas silvestres. Esse composto está relacionado com a diminuição nos níveis de marcadores inflamatórios principalmente do NF-kB, AP-1 e Nrf2, o que leva à redução de citocinas inflamatórias. Seu mecanismo se dá através da modulação da atividade de várias proteínas e fatores de transcrição, os quais estão envolvidos em vias metabólicas relacionadas à síntese de citocinas, quimiocinas e moléculas de adesão (SOUSA; PEREIRA, 2013).

O resveratrol pode atuar na prevenção de várias doenças, través da ativação do SIRT1, beneficiando o controle metabólico anormal, inflamação e defeitos do ciclo celular. Atua também na desacetilação de histonas e proteínas não-histonas, incluindo fatores de transcrição. Essa via sensibiliza o metabolismo, sobrevivência celular, resistência ao estresse oxidativo e imunidade (CHEN et al., 2015; BERMAN et al., 2017). 


\section{CHÁ VERDE}

O chá verde é obtido a partir da folha da planta Camelliasinensis, é rico em polifenóis pertencentes ao grupo dos flavonoides, mais especificamente as catequinas, como a epicatequina, a epicatequinagalato (ECG) e a epigalocatequina 3 galato (EGCG), que é a mais abundante. O chá verde apresenta cerca de 4.000 compostos bioativos e aproximadamente um terço desses compostos são polifenóis que são considerados os responsáveis pelos efeitos benéficos gerados como: os fatores anti-inflamatórios, antioxidantes, anticancerígenos, entre outros. (NAMITA; MUKESH; VIJAY, 2012). Haramizu e colaboradores (2013) demonstraram efeitos positivos da ingestão a longo prazo de catequinas do chá verde em ratos, atenuando os danos musculares induzidos pela corrida em declive, suprimindo a inflamação e acelerando a recuperação das fibras musculares.

Eichenberge e colaboradores (2010) examinaram os efeitos do consumo de três semanas de suplementação com extrato de chá verde (159 mg/dia de catequinas totais) no metabolismo e desempenho durante o ciclismo em atletas de endurance. Observou-se uma diminuição significativa da proteína $\mathrm{C}$ reativa (PCR), um marcador de inflamação que foi significativamente reduzido $(\mathrm{p}=0,045)$ após a suplementação em comparação ao placebo.

\section{QUERCETINA}

A quercetina representa cerca de $95 \%$ do total dos flavonoides ingeridos na dieta humana. A cebola, maçã e brócolis são as fontes majoritárias da quercetina. A quercetina possui a capacidade de inibição das proteínas ERK e JNK e suas formas fosforiladas. Em macrófagos, a quercetina suprime a transcrição do TNF- $\alpha$ por meio da diminuição da fosforilação e da ativação da JNK/SAPK, ao mesmo tempo que bloqueia a síntese de TNF- $\alpha$ por meio da inibição da fosforilação da ERK1/2, reduzem a resposta inflamatória por meio da redução da expressão gênica das enzimas COX-2 e iNOS, e pela diminuição da translocação do fator de transcrição NF-אB (BASTOS; ROGERO; ARÊA, 2009).

\section{GENGIBRE (GINGEROL)}

O gengibre é um tempero popular originário dos rizomas da planta Zingiberofficinale. Este rizoma tem sido utilizado como estratégia para o tratamento de diversas doenças, especialmente em culturas não ocidentais. A atividade anti-inflamatória do gengibre está relacionada com a capacidade de modular o linfócito T, diminuindo a eosinofilia, a quantidade de mastócitos, a liberação de IL-4 e a resposta Th2. Também pode levar a redução dos níveis de COX-2. Os componentes presentes no gengibre agem sobre o efeito de ativação do lipopolissacarídeo em macrófagos, levando a um efeito imunomodulador e anti-inflamatório. A principal via de sinalização envolvida é a do fator de 
transcrição de citocinas pró-inflamatórias NFkB, com participação das MAP quinases e ativação de transcrição Nrf2, relacionado a expressão de enzimas envolvidas nas defesas antioxidantes (VIEIRA et al., 2014; WILSON et al., 2015).

Zehsaz e colaboradores (2014) investigaram o efeito do gengibre nas concentrações plasmáticas de interleucina-1 $\beta$ (IL-1 $\beta$ ), interleucina-6 (IL-6) e fator de necrose tumoral- $\alpha$ (TNF- $\alpha$ ). A amostra foi composta de 28 corredores de alto nível de resistência, divididos em dois grupos (controle e experimental). Eles realizaram o mesmo volume semanal de treinamento por 12 semanas. O grupo que recebeu cápsulas de $500 \mathrm{mg}$ de gengibre em pó (três vezes ao dia durante o segundo período de 6 semanas) mostrou reduções significativas nas concentrações plasmáticas de IL-1 $\beta$, IL-6 e TNF- $\alpha$ em relação aos grupos controle.

Wilson e colaboradores (2015) evidenciaram que o gengibre pode atuar como um anti-inflamatório, reduzindo a ação da enzima ciclo-oxigenase-2 (COX-2), citosinas pró-inflamatórias e da síntese de leucotrienos e prostaglandinas.

\section{CONCLUSÃO}

Pode-se concluir que atletas se automedicam com frequência, utilizando anti-inflamatórios com o objetivo de diminuir a dor e melhorar lesões ocasionadas pela prática esportiva. Um fator agravante é a livre comercialização de alguns anti-inflamatórios e o uso indiscriminado de AINEs que desencadeia distúrbios sérios de saúde, principalmente dispepsias, problemas renais e problemas hepáticos, além de afetar negativamente a síntese proteica e consequentemente, o processo de hipertrofia e prejudicar a regeneração dos tecidos lesionados.

Os alimentos funcionais e plantas medicinais ricos em compostos bioativos podem atuar na prevenção e tratamento das dores e inflamações. Entre os alimentos e nutrientes mais estudados com potencial anti-inflamatório estão: cúrcuma, hesperidina, licopeno, alcaçuz, espinheira santa, o pequi, a cereja azeda, o ômega 3, resveratrol o chá verde, a quercetina e o gengibre. A utilização destes pode ser uma estratégia para a diminuição de dores e inflamações provocadas pelo exercício extenuante e uma resposta para a diminuição da administração de anti-inflamatórios que desencadeiam diversos problemas à saúde.

\section{REFERÊNCIAS}

ANDRADE, L. S.; MARREIRO, D. N. Aspectos sobre a relação entre exercício físico, estresse oxidativo e zinco. Revista de Nutrição Campinas, v. 24, n. 4, p. 629-640, 2011. Disponível em: https:// bit.ly/3pgBdsy 
ARAÚJO, M. A. Rodrigues. Hepatotoxicidade associada à nimesulida: uma revisão da literatura. Revista Brasileira de Farmacologia, v. 93, n. 3, p. 283-289, 2012. Disponível em: https://bit.ly/2JVxAYR

ATASHAK, S.; SHARAFI, H.; AZARBAYJANI, M. A.; STANNARD, S. R.; GOLI, M. A, HAGHIGH, M. M. Effect of omega-3 supplementation on the blood levels of oxidative stress, muscle damage and inflammation markers after acute resistance exercise in young athletes. Kinesiology, v. 45, n. 1, p. 22-29, 2013. Disponível em: https://hrcak.srce.hr/104544

BASTOS, D. H. M.; ROGERO, M. M.; ARÊAS, J. A. G. Mecanismos de ação de compostos bioativos dos alimentos no contexto de processos inflamatórios relacionados à obesidade. Arquivos Brasileiros de Endocrinologia \& Metabologia, v.53, n. 5, p. 646-655, 2009. Disponível em: https://bit.ly/3n5dLwg

BRAYNER, N. F.; SILVA, A. A.; ALMEIDA, F. R. de. O risco do uso irracional do paracetamol na população brasileira e seus efeitos na hemostasia. Revista Científica da Fasete, v. 18, n. 1, p. 6-9, jan. 2018. Disponível em: https://bit.ly/36nkZou

BELL, P. G. et al. Montmorency cherries reduce the oxidative stress and inflammatory responses to repeated days high-intensity stochastic cycling. Nutrients, v. 6, n. 2, p. 829-843, 2014. Disponível em: https://bit.ly/32sTQzk

BERMAN, A. Y.; MOTECHIN, R. A.; WIESENFELD, M, Y.; HOLZ, M. The therapeutic potential of resveratrol: a review of clinical trials. Precision Oncology, v. 1, n. 35, p. 2-9, 2017. Disponível em: https://go.nature.com/2JSKqqE

BRENOL J.C.T.; XAVIER, R.M.; MARASCA, J. Anti-inflamatórios não hormonais convencionais. Revista Brasileira de Medicina, 2000. Disponível em: https://bit.ly/36nlKy7.

BURUKOGLU, D. et al. Effects of Nonsteroidal Anti-Inflammatory Meloxicam on Stomach, Kidney, and Liver of Rats. Toxicology And Industrial Health, v. 32, n. 6, 2016. Disponível em: https://bit.ly/ $35 \mathrm{dTRJV}$

BUSHEL, P. R. et al. Blood gene expression signatures predict exposure levels. Proceedings Of The National Academy Of Sciences, v. 104, n. 46, p.18211-18216, 2007. Disponível em: https://bit.ly/ $32 \mathrm{t} 7 \mathrm{yma}$ 
CHANG, C.; LIN, H. Dysbiosis in gastrointestinal disorders. Best Practice\&ResearchClinical Gastroenterology, v. 30, n. 1, p. 3-15, fev. 2016. Disponível em: https://bit.ly/36hpzVz

CHEN, S.; XIAOLAN, Z. B.; RANA, L.; WANA, J.; XIAOFANG, W. B.; QINA, Y.; SHUA, F.; GAOA,Y.; YUANA, L.; ZHANGA, Q.; MANTIAN, M. Resveratrol improves insulin resistance, glucose and lipid metabolism in patients with non-alcoholic fatty liver disease: a randomized controlled trial. Digestive and Liver Disease, v. 47, n.1, p. 226-232, 2015. Disponível em: https://bit.ly/3lgXgfZ

CORREA, C. S.; BARONI, B.; CADORE, E. L. Ação ergogênica e utilização de anti-inflamatórios não-esteroidais e ibuprofeno no esporte. Efdeportes, Buenos Aires, v. 15, n. 144, 2010. Disponível em: https://bit.ly/35dNfL6

CORREA, C. S. et al. Efeito do uso profilático do anti-inflamatório não-esteroide ibuprofeno sobre o desempenho em uma sessão de treino de força. Revista Brasileira de Medicina do Esporte, v. 19, n. 2, p. 116-119, 2013. Disponível em: https://bit.ly/3pgCfEW

CORRIGAN, B.; KAZLAUSKAS, R. Medication Use in Athletes Selected for Doping Control at the Sydney Olympics (2000). Clinical Journal Of Sport Medicina, v. 13, n. 1, p. 33-40, 2003. Disponível em: https://bit.ly/3pbnzGU

DROBNIC, F. et al. Reductionofdelayedonsetmusclesorenessby a novel curcumin delivery system (Meriva): a randomised, placebo-controlledtrial. Journal of theInternationalSociety of Sports Nutrition, USA, v. 11, n. 31, p. s/p., 2014. Disponível em: https://bit.ly/2JT6Ckr

EICHENBERGER, P. et al. No effectsofthree-weekconsumptionof a greenteaextraction time trial performance in endurance-trainedmen. InternationalJournal for VitaminandNutritionResearch, v. 80, n. 1, p. 54-64, 2010. Disponível em: https://bit.ly/35e58cH

GARCIA, B. C, et al. Efeitos da dieta suplementada com ômega-3 no músculo sóleo de ratos submetidos à natação: análise histológica e morfométrica. Revista Brasileira de Medicina do Esporte, v. 16, n. 5, p. 363-367, 2010. Disponível em: https://bit.ly/3kiWCNQ

GARG, A. et. al. Chemistryandpharmacologyof the citrus bioflavonoidhesperidin. Phytotherapy Research, v. 15, p. 655-669, 2001. Disponível em: https://bit.ly/3pewJm8 
GARÓFOLO, A.; PETRILL, A. S. Balanço entre ácidos graxos ômega-3 e 6 na resposta inflamatória em pacientes com câncer e caquexia. Revista de Nutrição, v. 19, n. 5, p. 611-621, 2006. Disponível em: https://bit.ly/3kf6tEj

GRASSO, E. C.; AOYAMA, E. M.; FURLAN, M. R. Ação Anti-Inflamatória de Curcuma longa L. (ZINGIBERACEAE). Revista Eletrônica Thesis, v. 28, n. 1, p.117-129, 2017. Disponível em: https:// bit.ly/32Kq6OX

GILMAN; GOODMAN. As Bases Farmacológicas da Terapêutica. 11. Ed. Porto Alegre: Artmed, p. 1821, 2010.

GOMES, T. C. F. et al. Prevalência de automedicação envolvendo anti-inflamatórios em pacientes de pronto atendimento com diagnóstico prévio de hemorragia digestiva. Brazilian Journal of Health Review, v. 2, n. 4, p. 3077-3085, 2019. Disponível em: https://bit.ly/2JMktZD

HARAMIZU, S. et al. Catechins suppress muscle inflammation and hasten performance recovery after exercise. Med Sci Sports Exerc, v. 9, n. 45, p. 1694-702, 2013. Disponível em: https://bit.ly/ $2 \mathrm{GK} 05 \mathrm{Hv}$

HE, Y. Protective effects of 2,4-dihydroxybenzophenone against acetaminophen-induced hepatotoxicity in mice. World Journal Of Gastroenterology, Pleasanton, v. 17, n. 21, p. 2663-2666, 2011. Disponível em: https://bit.ly/38sVYuL

JAIN, D.; KATTI, N. Combination treatment of lycopene and hesperidin protect experimentally induced ulcer in laboratory rats. Journal Of Intercultural Ethnopharmacology, v. 4, n. 2, p.143-146, 2015. Disponível em: https://bit.ly/3lgeAC3

KERCKHOFFS, A. P. M.; AKKERMANS, L. M. A.; SMET, M. B. M. de; BESSELINK, M. G. H.; HIETBRINK, F.; BARTELINK, I. H.; BUSSCHERS, W. B.; SAMSOM, M.; RENOOIJ, W. Intestinal Permeability in Irritable Bowel Syndrome Patients: effects of NSAIDs. Digestive Diseases and Sciences, v. 55, n. 3, p. 716-723, 3 mar. 2010. Disponível em: https://bit.ly/35aYMe5

LARINI, L. Fármacos e Medicamentos. 1 ed. Porto Alegre: Artmed, 2008. 
LOPEZ-GARCIA, E.; SCHULZE, M. B.; MANSON, J. E.; MEIGS, J. B.; ALBERT, C. M.; RIFAI, N.; WILLETT, W. C.; HU, F. B. Consumption of (n-3) Fatty Acids Is Related toPlasma Biomarkers of Inflammation and Endothelial Activation in Women. Nutritional Epidemiology, v. 134, n. 7, p. 18061811, 2004. Disponível em: https://bit.ly/3kf82SO

LUCAS, G. N. C. et al.Pathophysiological aspects of nephropathy caused by non-steroidal anti-inflammatory drugs. Brazilian Journal Of Nephrology, v. 41, n. 1, p.124-130, 2019. Disponível em: https://bit.ly/3eFHAkb

MAHAN, L.K., ESCOTT-STUMP, S.; RAYMOND, J. Krause: alimentos, nutrição e dietoterapia.12 ed. Rio de Janeiro: Elsevier, 2012.

MAIO, R.; DICHI, J. B.; BURINI, R. C. Consequências nutricionais das alterações metabólicas dos macronutrientes na doença hepática crônica. Arquivos de Gastroenterologia, v. 37, n. 1, p. 52-57, 2000. Disponível em: https://bit.ly/3pnqLzs

MARTIN, C. A. et al. Ácidos Graxos Poli-insaturados Ômega-3 e Ômega-6: Importância e Ocorrência em Alimentos. Revista de Nutrição, v. 19, n. 6, p. 761-770, 2006. Disponível em: https://bit.ly/2JMkXip

MCANULTY, S. R. et al. Ibuprofen Use During Extreme Exercise: effects on oxidative stress and PGE2. Medicine \& Science in Sports \& Exercise, v. 39, n. 7, p. 1075-1079, 2007. Disponível em: https://bit.ly/35cNCFz

MILLION, M.; LAGIER, J.-c.; YAHAV, D.; PAUL, M.. Gut bacterial microbiota and obesity. Clinical Microbiology And Infection, Basileia, v. 19, n. 4, p. 305-313, abr. 2013. Disponível em: https://bit.ly/ $35 \mathrm{ggU} 6 \mathrm{x}$

MORITZ, B.; TRAMONTE, V. L. C. Biodisponibilidade do licopeno. Revista de Nutrição, v. 19, n. 2 , p. 265-273, 2006. Disponível em: https://bit.ly/2JVzfh3

MORO, M. G. et al. Effects of Selective Versus Non-Selective COX-2 Inhibition on Experimental Periodontitis. Brazilian Dental Journal, v. 30, n. 2, p.133-138, 2019. Disponível em: https://bit.ly/38nQuSd

NAMITA, P.; MUKESH, R.; VIJAY, K. J. Camelliasinensis (greentea): A review. Global JournalofPharmacology, v. 6, n. 2, p. 52-59, 2012. Disponível em: https://bit.ly/2U9FteV 
NWAICHI, E. O.; CHUKU, L. C.; OYIBO, N. J. Profile ofAscorbicAcid, Beta-CaroteneandLycopene in Guava, Tomatoes, HoneyandRedWine. InternationalJournalCurrentMicrobiologyApplied Science, v. 4, n. 2, p. 39-43, 2015. Disponível em: https://bit.ly/2IktZm6

O’CONNOR, S.; MCCAFFREY, N.; WHYTE, E.; MORAN, K.; LACEY, P. Nonsteroidal anti-inflammatory drug use, knowledge, and behaviors around their use and misuse in Irish collegiate student-athletes. The PhysicianAndSports Medicine, v. 47, n. 3, p. 318-322, 6 dez. 2018. Disponível em: https://bit.ly/2JSLta4

OLIVEIRA, A. L. M.; PELÓGIA, N. C. C. Cefaleia como principal causa de automedicação entre os profissionais da saúde não prescritores. Revista Dor, v. 12, n. 2, p. 99-103, 2011. Disponível em: https://bit.ly/3n9AOWU

OLIVEIRA, M. M. C. et al. O uso crônico de anti-inflamatórios não-esteroidais e seus efeitos adversos. Caderno de Medicina, v. 2, n. 2, p. 90-100, 2019. Disponível em: https://bit.ly/3kj6Cqd

PANAHI, Y. et al. Curcuminoid Treatment for Knee Osteoarthritis: a randomized double-blind placebo-controlled Trial. RevistaPhytother, v. 28, n. s/n., p. 1625- 1631, 2014. Disponível em: https://bit.ly/31f3Bsh

PANKANIN, E. Overreachingandovertrainingsyndrome - causes, symptoms, diagnostics and prevention. JournalofEducation, Health and Sport, Bydgoszcz, v. 8, n. 7, p. 424-429, 2018. Disponível em: https://bit.ly/2JJi4im

PREEDY, V. R.; WATSON, R. Tomatoes and Tomato Products: Nutritional, Medicinal and Therapeutic Properties. 1 ed. Science Publishers, Enfield, United States of America, p. 334-343. Jan. 2008. Disponível em: https://bit.ly/3kaJsm9

PUGGINA, E. F.; FILHO, H. T. MACHADO, D. R. L.; BARBANTI, V. J. Efeitos do treinamento e de uma prova de triathlon em indicadores de lesão muscular e inflamação. Revista Brasileira de Ciência do Esporte, v. 38, n. 2, p. 115-123, 2016. Disponível em: https://bit.ly/3n3869S

ROSE, E. H. et al. Uso referido de medicamentos e suplementos alimentares nos atletas selecionados para controle de doping nos Jogos Sul-Americanos. Revista Brasileira de Medicina do Esporte, v. 12, n. 5, 2006. Disponível em: https://bit.ly/3n90Idq 
RUDGARD, W. E.; HIRSCH, C. A.; COX, A. R. Amateur endurance athletes use of non-steroidal anti-inflammatory drugs: a cross-sectional survey: a cross-sectional survey. International Journal Of Pharmacy Practice, London, v. 27, n. 1, p. 105-107, 18 jul. 2018. Disponível em: https://bit.ly/31fAHZi

SANTO, P. R. et al. Supplementation of ômega-3 reduces delayed muscular pain after test of exhaustion in sedentary women. Revista Brasileira de Nutrição Esportiva, v. 1, n. 6, p. 01-10, 2007. Disponível em: https://bit.ly/3pcV2Rh

SHAMI, N. J. I. E.; MOREIRA, E. A. M. Licopeno como agente antioxidante. Revista Nutrição, v. 17, n. 2, p. 227-236, 2004. Disponível em: https://bit.ly/3pf7eBd

SILVA, C. S. et al. Consumo referido de medicamentos em atletas de uma universidade do sul do Brasil e sua relação com o estresse. Arquivos Catarinenses de Medicina, v. 45, n. 4, p. 41-52, 2016. Disponível em: https://bit.ly/32r7V0i

SILVA, L. M. et al. Hesperidin, a citrus flavanone glycoside, accelerates the gastric healing process of acetic acid-induced ulcer in rats. Chemico-biological Interactions, v. 308, n. 1, p.45-50, 2019. Disponível em: https://bit.ly/3ke6j0a

SILVA, L. S. et al. Incidência da automedicação no uso indiscriminado de anti-inflamatórios esteroidais e não esteroidais entre universitários de Imperatriz-MA. Brazilian Journal of Health Review, v. 2, n. 2, p. 862-887, 2019. Disponível em: https://bit.ly/35bjvyv

SILVA, M. I. A.; CHAVES, D. F. S. Alimentos antioxidantes em exercícios de endurance. Revista Brasileira de Nutrição Funcional, v. 15, n. 62, p. 8-15, 2015. Disponível em: encurtador.com.br/fmCER

SOUSA, M. S.; PEREIRA, M. C. A. Mecanismos moleculares de ação anti-inflamatória e antioxidante de polifenóis de uvas e vinho tinto na aterosclerose. Revista Brasileira de Plantas Medicinais, v. 15, n. 4, p. 617-626,2013. Disponível em: encurtador.com.br/zBIW7

SUETH - SANTIAGO, S. V. et al. Curcumina, o pó dourado do açafrão-da-terra: Introspecções sobre química e atividades biológicas. Química Nova, v. 38, n. 4, p. 538-552, 2015. Disponível em: encurtador.com.br/tBIZ1

TAKAHASHI, M. et al. EffectsofCurcuminSupplementationonExercise-InducedOxidative Stress in Humans. International Journalof Sports Medicine, v. 35, n. 06, p. 469-475, 2014. Disponível em: encurtador.com.br/aoIO6 
TSITSIMPIKOU, C., KIOUKIA-FOUGIA, N., TSAROUHAS, K., STAMATOPOULOS, P., RENTOUKAS, E., KOUDOUNAKOS, JAMURTAS, A. Administrationoftomatojuiceameliorateslactatedehydrogenaseandcreatininekinase responses toanaerobic training. Food andchemicaltoxicology, 61, 9-13. 2013. Disponível em: encurtador.com.br/yAHN6

VIEIRA, N. A. et al. Efeito anti-inflamatório do gengibre e possível via de sinalização. Ciências Biológicas e da Saúde, v. 35, n. 1, p. 149-162, 2014. Disponível em: encurtador.com.br/msCZ3

VILELA, P. C. T. Perturbações Fisiológicas Induzidas pelos Anti-Inflamatórios Não Esteroides: a problemática da banalização do acesso ao medicamento. Universidade de Coimbra, 2019. Disponível em: encurtador.com.br/lqEH1

VITALE, K. C.; HUEGLIN, S.; BROAD, E. Tart Cherry Juice in Athletes. Nutrition and Ergogenic Aids, Michigan, v. 16, n. 4, p. 230-239, 2017. Disponível em: encurtador.com.br/gj116

XAVES, G. T. et al. Ação do ibuprofeno na regeneração muscular. Revista Conexão Eletrônica, Três Lagoas, v. 14, n. 1, p. 1524-1535, 2017. Disponível em: encurtador.com.br/nwzW0

WILSON, P. B. Ginger (Zingiberofficinale) as analgesicandergogenic aid in sport: a systemic review. The Journal of Strength and Conditioning Research, v. 29, n. 10, p.2980-95, 2015. Disponível em: encurtador.com.br/lFGWY

ZEHSAZ, F.; FARHANGI, N.; MIRHEIDARI,L. The effectofZingiberofficinale R. rhizomes (ginger) on plasma pro-inflammatorycytokine levels in well-trained male endurancerunners. Central European Journal of Immunology, v. 39, n. 1, p. 174-180, 2014. Disponível em: encurtador.com.br/betyE 